Revue d'histoire de l'Amérique française

REVUE D.HISTOIRE DE L'AMÉRIQUE FRANÇAISE

\title{
Liste des thèses présentées à l'Institut d'histoire (Université de Montréal, faculté des lettres) 1947-1965
}

Volume 20, numéro 3, décembre 1966

URI : https://id.erudit.org/iderudit/302611ar

DOI : https://doi.org/10.7202/302611ar

Aller au sommaire du numéro

Éditeur(s)

Institut d'histoire de l'Amérique française

ISSN

0035-2357 (imprimé)

1492-1383 (numérique)

Découvrir la revue

Citer ce document

(1966). Liste des thèses présentées à l'Institut d'histoire (Université de Montréal, faculté des lettres) 1947-1965. Revue d'histoire de l'Amérique française, 20(3), 515-521. https://doi.org/10.7202/302611ar d'utilisation que vous pouvez consulter en ligne. 


\section{UNIVERSITE DE MONTREAL}

\section{FACUITE DES LETTRES}

\section{LISTE DES THESES PRESENTÉES À L'INSTITUT D'HISTOIRE (1947-1965)}

N.B. - Le mot "manque" indique que le volume n'est pas au département d'histoire.

\section{I - THESES : MAITRISES}

\section{A. (1947)}

1. Gagné, Lucien, c.s.s.r., Etudes des sources sur CharlesMichel de Salaberry (1778-1810).

$$
\text { M. A. (1949) }
$$

2. Léger, Jean-Marc, Les répercussions politiques et sociales sur les Canadiens de l'invasion par les insurgés des treize colonies, en 1775.

3. Tessier, Florent G., Frontenac et l'eau-de-vie (1672-1682).

4. Vigneau, Roger, Le problème historique des origines franciscaines.

M. A. (1950)

5. Arbour, Colette, La diplomatie iroquoise.

6. Bertrand, Maurice, La formation des Gaules. (Manque).

7. Forget, Eucher, s.j., Louis Riel et les troubles de la Rivière Rouge.

8. Gingras, Gérard, Le culte des empereurs romains.

9. Marie-de-Saint-Jean d'Ars, (Simone Charette), c.s.c., La carte des Jésuites du lac Supérieur. (Manque).

M. A. (1951)

10. Bousquet, Denis, Le Canada et la guerre des brochures.

11. Celovski, Boris, La méthode de la science de l'histoire: esquisse de son évolution, jusqu'à Ernst Bernheim. 
12. Choplin, Robert, Un mouvement français d'expansion coloniale au XVIIe siècle: la fondation de Montréal.

13. Gauthier, Pierre, L'idée de Rome impériale chez Virgile. (Manque).

14. Lamontagne, Roland, L'eau-de-vie en Nouvelle-France (16291672).

15. Ravary, Viateur, c.s.v., La vérité historique dans l'œuvre du Baron de Lahontan. (Manque).

16. Samuel (Benoit Charette), f.s.g., Monographie du village de l'Annonciation.

17. Weilbrenner, Bernard, Introduction aux voyages des La Verendrye.

M. A. (1952)

18. Adélard-Marie (Camille Mercure), f.s.g., Monseigneur Bourget, premières années d'épiscopat, 1837-1842.

19. Bégin, Joseph-Octave, s.j., Le Père Jérôme Lalemant, s.j., supérieur de la mission huronne (1638-1645).

20. Dominique-Marie (Georges Delisle), f.s.g., Les Frères hospitaliers de la Croix et de Saint-Joseph et leurs démarches en France (1717-1734).

21. Gagnon, abbé Paul, Trois étapes du césaropapisme.

22. Hardie, Thomas, Céloron de Blainville.

23. Héroux, Maurice, L'opinion canadienne devant la guerre de 1914.

24. Marcel-Joseph (Georges-Etienne Baillargeon), f.s.g., Les Canadiens veulent conserver le régime seigneurial (18001826).

25. Rouleau, Jean-Noël, La société canadienne au XVIIIe siècle, d'après le témoignage de Madame Bégon.

\section{A. (1953)}

26. Amédée-Joseph (Lucien Boụchard), f.s.g., L'Histoire $d u$ Canada de Garneau: étude comparée de la première et de la cinquième éditions.

27. Bonardelli, Peter M., Canada and the Atlantic Pact.

28. Forget, Hélène, L'agitation sentinelliste au Rhode-Island (1924-1929).

29. François-Georges (Jean Laprotte), f.i.c., Aperçus statistiques sur le Bas-Canada, d'après les Journaux de la Chambre d'Assemblée (1793-1835). 
30. Giguère, Georges-Emile, s.j., Une édition à Québec en 1858 : Les relations des Jésuites.

31. Pierre Nolasque (Jules Sawyer), f.i.c., Index des documents contenus dans les Rapports des Archives publiques du Canada.

32. Potvin, Fernand, s.j., Saint Antoine Daniel, premier martyr canadien.

33. Surprenant, André, s.j., Le Père Pierre-Joseph-Marie Chaumonot, missionnaire de la Huronie.

M. A. (1954)

34. Ernest-Joseph (Hector Lévesque), f.i.c., Lévis au Canada (1756-1760).

35. Lavallée, abbé Germain, Monseigneur Antoine Racine dans la question universitaire canadienne (1874-1893).

36. Vinet, Bernard, François-Pierre Rigaud de Vaudreuil (17031779).

\section{A. (1955)}

37. Côté, Jean, s.j., L'institution des donnés à Sainte-Marie-desHurons.

38. Mondou, abbé Paul A., Une autobiographie au XIIe siècle: le De Vita Sua de Guibert de Nogent. (Manque).

39. Vanasse, Alfred R., On the Durham Report: The FrenchEnglish Relationship as contained in the Durham Report and how subsequent historians have interpreted it.

M. A. (1956)

40. Bùi Duc-Sinh, o.p., Aspects de l'évolution littéraire et scientifique au Vietnam.

41. Euclide (René Desrosiers), f.c., La Congrégation des frères de la Charité s'implante définitivement à Montréal (18651870).

42. Mercier, abbé Jean, L'Estrie: ses limites, son onomastique.

43. Painchaud, Paul, Le service civil de la province de Québec (1867-1900).

\section{A. (1957)}

44. Chaussé, Gilles, s.j., Le Père Paul Le Jeune, missionnairecolonisateur.

45. Lapointe, André, Aventures politiques et judiciaires de l'Acte de l'Amérique du Nord Britannique de 1880-1884. (Manque). 
46. Wallot, Jean-Pierre, La querelle des prisons (1805-1807). M. A. (1958)

47. André-Julien (André Sénécal), f.i.c., Les textes essentiels du journal L'Avenir (1847-1858).

48. Bernard, Jean-Paul, La pensée des journalistes libéraux de Saint-Hyacinthe (1853-1864).

49. Brien, André, c.s.v., F.-M.-F. Ruette d'Auteuil, procureur du Conseil souverain, (1659-1737).

50. Gabriel-Marie (Berthe Legault), s.s.a., Histoire de la paroisse Sainte-Geneviève (1741-1872).

51. Gougeon, Jacques, L'affaire de Suez et le Canada.

52. Guimond, Lionel, La Gazette de Montréal de 1785 à 1790.

M. A. (1959)

53. Jolicœur, Louis-Philippe, L'Action française de Montréal (1917-1928): étude suivie d'un index.

54. Lefebvre, Esther, r.h.s.j., Marie Morin, premier historien canadien de Ville-Marie.

55. Marie-de-Saint-Albert-du-Carmel (Hélène Desjardins), c.s.c., La correspondance de Pline le Jeune, miroir d'une société.

56. Nish, James Cameron, Inventaire de la correspondance publique d'Henri Bourassa (1895-1924).

M. A. (1960)

57. Ausserleitner, Walter Joseph, Soviet Ideological Penetration of Central Germany (1945-1957).

58. Brosseau, Jean-Luc (Gérard Brosseau), f.i.c., L'Amérique, nouveau champ apostolique des Frères de l'Instruction chrétienne.

59. Girard, Mathieu, Jules-Paul Tardivel, rédacteur en chef et propriétaire de La Vérité.

60. Legris, Claude, Entrée du Canada sur la scène internationale et ses nouvelles relations avec les Etats-Unis (1919-1927). (Manque).

61. Le Scouarnec, Jean-Louis, De l'existence de l'Ecole du Palais sous les Mérovingiens et sous les Carolingiens.

62. Marc-Henri (Joseph Sarrazin), f.s.c., Projets de restauration par le corporatisme (1933-1950).

63. Tousignant, Pierre, La Gazette de Montréal de 1791 à 1796. 
64. Valois, abbé Charles, La Chambre d'Assemblée du BasCanada (1792-1815).

\section{A. (1961)}

65. Bernier, abbé Gilles, Les idées colonisatrices de l'intendant Jacques de Meulles.

66. Herrnritt, Dora, The Political Career of T. G. Masaryk.

67. Vallerand, Noël, Berlin, 1945-1949: point de rencontre des illusions occidentales et des ambitions soviétiques.

68. Zoltvany, Yves-Francois, Les libéraux du Québec, leur parti et leur pensée (1867-1873).

M. A. (1962)

69. Arbour, Henri (Jean-Pierre Arbour), f.i.c., Le bill des notables, 1831.

70. Leblanc, André, The Defeat of the Cartier-Macdonald Ministry and the Militia Bill of 1862.

71. Robichaud, Emile, La Gazette des Trois-Rivières (18171820); le Spectateur canadien (1815-1820).

M. A. (1963)

72. Bernard, Claude, Les Juifs dans l'Etat franc durant le Haut Moyen Age (500-840).

73. D'Allaire, Micheline, La monarchie et l'unification de la France au XVIIe siècle.

74. Saint-Germain, Yves, La prospérité nord-américaine de l'après-guerre: le Québec et les Canadiens français (19191927).

75. Leduc, abbé Pierre, Les origines et le développement de l'art association de Montréal (1860-1912).

M. A. (1965)

76. Dupont, Antonin, Quelques problèmes politiques et sociaux (1933-1935) d'après l'Action Nationale, le Canada et le Devoir.

77. Poulin, Hughes, Les Institutions politiques de l'Angleterre sous les Anglo-Saxon's et les Normands (600-1154).

78. Touchette, Claude, L'Administration de Lord Sydenham, 1839-1841, d'après sa correspondance officielle avec Lord Russell.

79. Tourangeau, André, L'Opinion du Devoir sur les événements importants de l'actualité 1912 à 1914. 


\section{II - MEMOIRES PREDOCTORAUX}

Bertrand, Denis, Les impératifs de la politique internationale et militaire canadienne (1935-1939).

Deshaies, Bruno, Deux législatures provinciales: Québec et Ontario (1867-1868).

Falmagne, Jacques, L'expansion grecque du XIIe siècle au XVIe siècle avant Jésus-Christ.

Grenon, Michel, La Hollande et la révolution intellectuelle des $X V I I$ e et XVIIIe siècles.

Lefebvre, André, Les Montrealers et la crise politique du BasCanada (juillet 1835 - mars 1836).

\section{III - MÉMOIRE DU DIPLOME D'ETUDES SUPERIEURES}

Giry, Danielle, L'apparition des professions bancaires dans l'Occident médiéval (Ve-XIIe siècle).

\section{IV - THESES : DOCTORATS}

1. Séguin, Maurice, D. ès L. (1947), La nation canadienne et l'agriculture (1760-1850).

2. Michaud, Marguerite M., D. ès A. (1947), La reconstruction française au Nouveau-Brunswick.

3. Gagné, Lucien, c.s.s.r., D. ès L. (1948), Salaberry (17781829).

4. Comeau, Joseph-Edouard, D. ès A. (1949), L'enseignement du français dans les écoles publiques de la Nouvelle-Ecosse depuis 1900.

5. Emery, Jules, s.j., D. ès A. (1949), Mémoires touchant saint Isaac Jogues.

6. Larivière, Florian, s.j., D. ès A. (1950), Vie de Saint Charles Garnier.

7. Latourelle, René, s.j., D. ès A. (1950), Etude sur les écrits de Saint Jean-de-Brébeuf.

8. Lamontagne, Roland, Ph. D. (1953), Jean Talon et la colonisation de la Nouvelle-France.

9. Marie-de-Saint-Jean-d'Ars (Simone Charette), c.s.c., Ph.D. (1953), Claude Dablon, s.j., et la Nouvelle-France (16551697). 
10. Bousquet, Denis, $\mathrm{Ph} . \mathrm{D}$. (1954), Les conférences impériales et la création du troisième Empire britannique (1887-1931).

11. Bilodeau, Rosario, D. ès L., (1956), Liberté économique et politique des Canadiens sous le régime français.

12. Hardie, Thomas, Ph. D. (1957), Claude de Ramezay.

13. Kage, Joseph, $\mathrm{Ph}$. D. (1958), Jewish Immigration and Immigrant Aid Effort in Canada (1760-1957).

14. Vanasse, Alfred R., F'h. D. (1958), A Social History of the Seigniorial Regime in Canada (1712-1739).

15. Mondou, abbé Paul A., Ph. D. (1959), Guibert de Nogent (1053-1124) et la continuité historique au XIIe siècle.

16. Prince-Falmagne, Thérèse, D. ès L. (1959), Un gouverneur de la Nouvelle-France: Jacques de Brisay, marquis de Denonville.

17. Falmagne, Jacques, D. ès L. (1961), Beaudoin V, comte de Hainaut et de Flandre, marquis de Namur (1150-1195).

18. Ausserleitner, Walter J., Ph. D. (1962), The Double Dilemma of Contemporary German Communist Historiography.

19. Sevenster, Gelske, Ph. D. (1962), La vie et l'œuvre de Jacques-Cartier.

20. Marcel-Joseph (Georges-Etienne Baillargeon), f.s.g., Ph. D. (1963), L'abolition du régime seigneurial (1820-1854).

21. Giguère, Georges-Emile, s.j., Ph. D. (1965), La Restauration de la Compagnie de Jésus au Canada (1839-1857).

22. Wallot, Jean-Pierre, Ph. D. (1965), Le Bas-Canada sous l'administration de Craig (1807-1811).

23. Bertrand, Denis, D. ès L. (1965), La politique extérieure et militaire du Canada et la réaction canadienne-française d̀ la veille de la deuxième grande guerre (1935-1939). 